\title{
Torical Modification of Newton non-degenerate ideals.
}

\author{
Fuensanta Aroca* \\ (Universidad Nacional Autónoma de México.) \\ Mirna Gómez-Morales. ${ }^{\dagger}$ (University of Warwick.) \\ Khurram Shabbir ${ }^{\ddagger}$ \\ (Department of Mathematics, GC University Lahore.)
}

December 3, 2018

Dedicated to professor Hironaka in his 80th birthday.

\section{Introduction.}

Newton non degenerate singularities were introduced in the 70's Ehl, Kou, Kho2. For hypersurfaces, the definition is given in terms of the Newton Polyhedron of the function defining the hypersurface. For complete intersection singularities they are characterized in terms of the Newton polyhedra of a given set of generators of the ideal. The reference book on the subject is Oka2.

Newton non degenerate singularities of hypersurfaces and of complete intersections have been widely studied (See for example [BrNe, $\mathrm{Kou}$ ] and $\mathrm{Kho1}$, Oka1, Biv, $\mathrm{SaZu}]$ ). A good resolution of a non degenerate singularity may be constructed from the dual fan of the Newton boundaries.

In this paper we extend the definition of Newton non degenerate to non necessarily complete intersection singularities. Our definition is new and does not depend on the system of generators chosen and is given in terms of initial ideals.

The Gröbner fan of an ideal is the extension to non-principal ideals of the concept of fan dual to the Newton polyhedron. The tropical variety associated to a hypersurface $H$ is the $(\operatorname{dim} H-1)$-skeleton of the fan dual to the Newton polyhedron of the function defining the hypersurface. Reference books on Tropical Geometry are Gat, IMS, RiSt.

\footnotetext{
*Research partially supported by PAPIIT-UNAM, CONACYT (Mexico) grants 55084, 162340 and 117110

${ }^{\dagger}$ Research supported CONACYT (Mexico) grant 213186

${ }^{\ddagger}$ Partially supported by CONACYT (Mexico) and TWAS (Italy) grant FR 3240223595. The third author wants to thank these institutions for their support.
} 
In [AIL] an extension of the Newton-Puiseux method to compute parametrizations of plane curves is extended to arbitrary codimension replacing the Newton Polyhedron by the tropical variety.

We prove that a regular refinement of the Gröbner fan of the ideal defining the non degenerate variety gives a resolution. We also prove that the strict transform intersects the orbit associated to a cone if and only if the cone is contained in the tropical variety. Both results are original statements.

The second author would like to thank Carles Bibia-Ausina and Dmitry Kerner, for clarifying discussions on the subject and, to Meral Tosun, for the example in section 11. The third author would like to thank A. Jensen for answering several questions by e-mail. In particular for the proof of Proposition 10.6 .

\section{Cones and fans.}

In this section we introduce some basic concepts of convex geometry. These concepts may be found in several books (see for example [Ful]).

Given vectors $u^{(1)}, \ldots, u^{(k)} \in \mathbb{R}^{n}$. The polyhedral cone generated by $u^{(1)}, \ldots, u^{(k)}$ is the set

$$
\sigma=\left\langle u^{(1)}, \ldots, u^{(k)}\right\rangle:=\left\{\lambda_{1} u^{(1)}+\cdots+\lambda_{k} u^{(k)} ; \lambda_{i} \in \mathbb{R}_{\geq 0}, i=1, \ldots, k\right\} \subset \mathbb{R}^{n} .
$$

The vectors $u^{(i)}$ 's are called the generators of the cone. A polyhedral cone is said to be rational if it has a set of generators in $\mathbb{Z}^{n}$.

We will denote by $e^{(1)}, \ldots, e^{(n)}$ the vectors in the standard base of $\mathbb{R}^{n}$. With this notation the first orthant is the cone $\left(\mathbb{R}_{\geq 0}\right)^{n}=\left\langle e^{(1)}, \ldots, e^{(n)}\right\rangle$.

The cone generated by the columns of a matrix $M$ will be denoted by $\langle M\rangle$.

The dimension of $\sigma$ is the dimension of the minimal linear subspace $\mathcal{L}(\sigma)$ containing $\sigma$ and is denoted by $\operatorname{dim}(\sigma)$. The dimension of $\langle M\rangle$ is equal to the rank of the matrix $M$.

The relative interior of a cone $\sigma$ is the interior of $\sigma$ as a subset of $\mathcal{L}(\sigma)$. That is

$$
\operatorname{Int}_{r e l}\left\langle u^{(1)}, \ldots, u^{(s)}\right\rangle=\left\{\lambda_{1} u^{(1)}+\cdots+\lambda_{s} u^{(s)} ; \lambda_{i} \in \mathbb{R}_{>0}\right\} .
$$

The dual $\sigma^{\sqrt{ }}$ of a cone $\sigma$ is the cone given by

$$
\sigma^{\sqrt{ }}:=\left\{v \in \mathbb{R}^{n} ; v \cdot u \geq 0, \text { for all } u \in \sigma\right\}
$$

where $u \cdot v$ stands for the inner product $u \cdot v:=u_{1} v_{1}+\cdots+u_{n} v_{n}$ of the vectors $u=\left(u_{1}, \ldots, u_{n}\right)$ and $v=\left(v_{1}, \ldots, v_{n}\right)$.

Let $M$ be an unimodular matrix. We have

$$
\langle M\rangle^{\vee}=\left\langle\left(M^{-1}\right)^{t}\right\rangle
$$

where $M^{t}$ stands for the transpose of the matrix $M$. 
A rational polyhedral cone is said to be strongly convex if it does not contain any non-trivial linear subspace. A cone contained in the first orthant is strongly convex. The dual of a cone of maximal dimension is strongly convex.

A vector in $\mathbb{Z}^{n}$ is said to be primitive when the maximum common divisor of its coordinates is 1 .

The set of vectors $\left\{u^{(1)}, \ldots, u^{(k)}\right\} \subset \mathbb{Z}^{n}$ is the set of vertices of a rational strongly convex cone $\sigma$ when

- $u^{(i)}$ is primitive for $i \in\{1, \ldots, k\}$.

- $\sigma=\left\langle u^{(1)}, \ldots, u^{(k)}\right\rangle$.

- $\left\langle u^{(1)}, \ldots, u^{(i-1)}, u^{(i+1)}, \ldots, u^{(k)}\right\rangle \subsetneq \sigma$ for $i=1, \ldots, k$.

By $\sigma=\operatorname{Cone}\left(u^{(1)}, \ldots, u^{(k)}\right)$ we will denote the rational convex cone with vertices $u^{(1)}, \ldots, u^{(k)}$. We will also write $\sigma=\operatorname{Cone}(M)$ where $M$ is the matrix that has as columns the vertices of $\sigma$.

A strongly convex rational polyhedral cone $\sigma=\operatorname{Cone}\left(u^{(1)}, \ldots, u^{(k)}\right)$ is said to be regular when the group $\mathcal{L}(\sigma) \cap \mathbb{Z}^{n}$ is of rank $k$ and is generated by the vertices of $\sigma$.

Remark 2.1. The vertices of a regular cone are linearly independent over $\mathbb{Q}$. An $n$-dimensional rational cone in $\mathbb{R}^{n}$ is regular if and only if $\sigma=\operatorname{Cone}(M)$ where $M \in G L(\mathbb{Z}, n)$ is an unimodular matrix.

Remark 2.2. Let $\sigma=\operatorname{Cone}\left(u^{(1)}, \ldots, u^{(k)}\right)$ be a regular cone. The faces of $\sigma$ are the cones Cone $\left(u^{\left(i_{1}\right)}, \ldots, u^{\left(i_{s}\right)}\right)$ with $\left\{i_{1}, \ldots, i_{s}\right\} \subset\{1, \ldots, k\}$.

A collection of cones $\Sigma$ in $\mathbb{R}^{n}$ is called a polyhedral fan if it satisfies the following properties:

i) Every face of a cone in $\Sigma$ is a cone in $\Sigma$;

ii) The intersection of any two cones $\sigma, \tau \in \Sigma$ is a face of both $\sigma$ and $\tau$.

A polyhedral fan is said to be regular if all of its cones are regular.

Remark 2.3. Let $\Sigma$ be a regular fan and let $\tau$ and $\sigma$ be cones in $\Sigma$. By remark 2.2 the cone $\tau$ is a face of $\sigma$ if and only if the set of vertices of $\tau$ is a subset of the set of vertices of $\sigma$. That is $\tau=\operatorname{Cone}(T)$ and $\sigma=\operatorname{Cone}(M)$ where $M=(T, S)$.

A fan $\Sigma^{\prime}$ is a refinement of a fan $\Sigma$ if every $\sigma \in \Sigma$ is a union of cones in $\Sigma^{\prime}$. A refinement $\Sigma^{\prime}$ is called regular if every cone in $\Sigma$ is regular.

Proposition 2.4. Any fan has a regular refinement.

The proof is left as an exercise in section 2.6 of [Ful. 


\section{Newton Polyhedron.}

Let $\mathbb{K}$ be an algebraically closed field of any characteristic.

A polynomial in $n$ variables with coefficients in $\mathbb{K}$ is an expression of the form

$$
f(x)=\sum_{\mu \in \Omega \subset \mathbb{Z}_{\geq 0}^{n}} a_{\mu} x^{\mu} ; \# \Omega<\infty, a_{\mu} \in \mathbb{K}
$$

where $x^{\mu}:=x_{1}^{\mu_{1}} \cdots x_{n}^{\mu_{n}}$.

The ring of polynomials in $n$ variables with coefficients in $\mathbb{K}$ will be denoted by $\mathbb{K}\left[x_{1}, \ldots, x_{n}\right]$.

The support or set of exponents of $f$ is defined by

$$
\varepsilon(f):=\left\{\mu \in \mathbb{Z}_{\geq 0}{ }^{n} ; a_{\mu} \neq 0\right\} .
$$

Remark 3.1. Given $f \in \mathbb{K}\left[x_{1}, \ldots, x_{n}\right]$, as in $(2)$, we have $f(\underline{0})=a_{\underline{0}}$, and, then,

$$
f(\underline{0}) \neq 0 \Longleftrightarrow \underline{0} \in \varepsilon(f) .
$$

The Newton polyhedron of $f \in \mathbb{K}\left[x_{1}, \ldots, x_{n}\right]$ is the convex hull

$$
N P(f):=\operatorname{Conv}\left(\left\{\mu+\left(\mathbb{R}_{\geq 0}\right)^{n} ; \mu \in \varepsilon(f)\right\}\right) .
$$
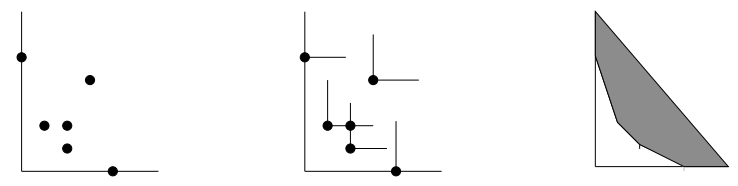

Figure 1: $\varepsilon(f), \varepsilon(f)+\mathbb{R}_{\geq 0}^{2}, N P(f)$

Remark 3.2. Given $f \in \mathbb{K}\left[x_{1}, \ldots, x_{n}\right]$, by Remark 3.1, $f(\underline{0}) \neq 0$ if and only if the Newton polyhedron of $f$ is the first orthant.

Remark 3.3. Let $f$ be a polynomial in $\mathbb{K}\left[x_{1}, \ldots, x_{n}\right]$. The Newton polyhedron of $f$ has only one vertex if and only if

$$
f=x^{\alpha} h
$$

where $h$ is a polynomial in $\mathbb{K}\left[x_{1}, \ldots, x_{n}\right]$ with $h(\underline{0}) \neq 0$, and $\alpha$ are the coordinates of the vertex of $N P(f)$.

Let $f$ be a polynomial as in 2 and let $F$ be a face of the Newton polyhedron of $f$. The restriction of $f$ to the set $F \subset \mathbb{Z}^{n}$ is defined as

$$
\left.f\right|_{F}:=\sum_{\mu \in \varepsilon(f) \cap F \subset \mathbb{Z}^{n}} a_{\mu} x^{\mu} .
$$

Remark 3.4. Let $f$ be a polynomial in $\mathbb{K}\left[x_{1}, \ldots, x_{n}\right]$. We have

$$
\left.f\right|_{\left\langle e^{(1)}, \ldots, e^{(s)}\right\rangle}=f\left(x_{1}, \ldots, x_{s}, 0, \ldots, 0\right) .
$$




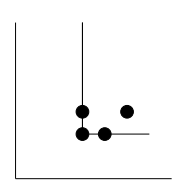

Figure 2: $N P(f)$,

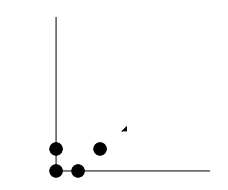

$N P(h)$ where $f=x^{3} y^{2} h$

\section{The dual fan.}

Given $\omega \in\left(\mathbb{R}_{\geq 0}\right)^{n}$ the $\omega$-order of a non-zero polynomial in $\mathbb{K}\left[x_{1}, \ldots, x_{n}\right]$ is defined as

$$
\nu_{\omega}(f):=\min \{\omega \cdot \mu ; \mu \in \varepsilon(f)\} .
$$

Let $f \in \mathbb{K}\left[x_{1}, \ldots, x_{n}\right]$ be a polynomial. Given a vector $\omega$ in the first orthant set

$$
\pi_{\omega}(f):=\left\{x \in \mathbb{R}^{n} ; \omega \cdot x=\nu_{\omega}(f)\right\} .
$$

The hyperplane $\pi_{\omega}(f)$ is a supporting hyperplane for $N P(f)$.
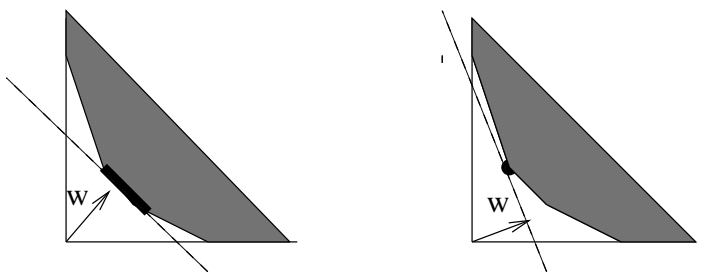

Figure 3: face $_{w}(f)$

The intersection

$$
\operatorname{face}_{\omega}(f):=\pi_{\omega}(f) \cap N P(f)
$$

is a face of $N P(f)$.

Given a face $F$ of $N P(f)$ set

$$
C_{F}:=\left\{\omega \in\left(\mathbb{R}_{\geq 0}\right)^{n} ; F \subset \text { face }_{\omega}(f)\right\} .
$$

The collection of cones

$$
\Sigma(f):=\left\{C_{F} ; F \text { is a face of } N P(f)\right\}
$$



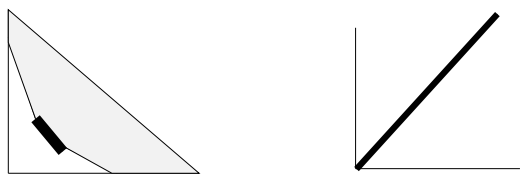

Figure 4: $F, C_{F}$

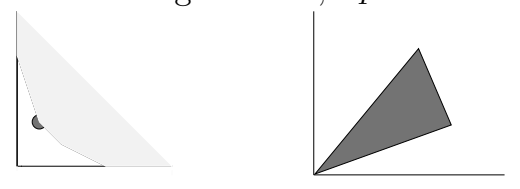

Figure 5: $\mathbf{P}, C_{\mathbf{P}}$

forms a fan.
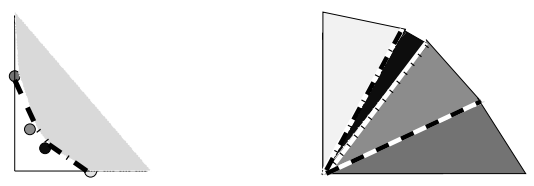

Figure 6:

Remark 4.1. Given $f \in \mathbb{K}\left[x_{1}, \ldots, x_{n}\right]$, if $f(\underline{0}) \neq 0$ then, by remark 3.2 , we have

$$
\Sigma(f)=\left\{\left\langle e^{\left(i_{1}\right)}, \ldots, e^{\left(i_{s}\right)}\right\rangle ;\left\{i_{1}, \ldots, i_{s}\right\} \subset\{1, \ldots, n\}\right\} .
$$

4

The mapping

$$
\begin{array}{ccc}
\{\text { faces of } N P(f)\} & \longrightarrow & \Sigma(f) \\
F & \mapsto & C_{F}
\end{array}
$$

gives a duality.

For $\omega$ in the relative interior of the cone $C_{F}$ we have face $_{\omega}(f)=F$.

Remark 4.2. Given $\omega$ and $\omega^{\prime}$ in the relative interior of the cone $\left\langle e^{\left(i_{1}\right)}, \ldots, e^{\left(i_{s}\right)}\right\rangle$. The equality face $_{\omega} f=$ face $_{\omega^{\prime}} f$ holds if and only if $\omega$ and $\omega^{\prime}$ belong to the relative interior of the same cone of $\Sigma(f)$.

Remark 4.3. Let $\mathbf{P}$ be a vertex of $N P(f)$. We have

$$
\varepsilon(f) \subset \mathbf{P}+C_{\mathbf{P}} \sqrt{ } .
$$

Definition 4.4. We will say that $\sigma$ is a good cone for $f$ if it is contained in a cone of $\Sigma(f)$. 

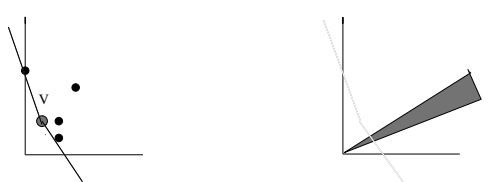

Figure 7: $\mathbf{P}, C_{\mathbf{P}}$

Let $f \in \mathbb{K}\left[x_{1}, \ldots, x_{n}\right]$ be a polynomial and let $\sigma$ be a good cone for $f$. We have that ace $_{\omega} f=$ face $_{\omega^{\prime}} f$ for $\omega, \omega^{\prime} \in I_{n+l} \sigma$. We define the $\sigma$-face of $f$ as

$$
\text { face }_{\sigma} f:=\text { face }_{\omega} f \quad \text { where } \omega \in \operatorname{Int}_{\text {rel }}(\sigma) .
$$

Remark 4.5. Let $\sigma$ be a good cone for $f$ of maximal dimension. We have

$$
\varepsilon(f) \subset \mathbf{P}+\sigma^{\vee}
$$

where $\mathbf{P}$ is the vertex dual to the cone of $\Sigma(f)$ containing $\sigma$.

\section{Monomial transformations}

Given an unimodular matrix with integer entries, $M \in G L(n, \mathbb{Z})$, we will denote by $\phi_{M}$ the morphism given by

$$
\begin{aligned}
\phi_{M}:\left(\mathbb{K}^{*}\right)^{n} & \longrightarrow\left(\mathbb{K}^{*}\right)^{n} \\
z & \longmapsto\left(z^{u^{(1)}}, z^{u^{(2)}}, \ldots, z^{u^{(n)}}\right)
\end{aligned}
$$

where $u^{(1)}, u^{(2)}, \ldots, u^{(n)}$ are the columns of the matrix $M$ and $\mathbb{K}^{*}:=\mathbb{K} \backslash\{0\}$.

The morphism $\phi_{M}$ is bi-rational on $\mathbb{K}^{n}$. It is bi-regular on the torus $\left(\mathbb{K}^{*}\right)^{n} \simeq$ $\mathbb{T}^{n}$ and the following equalities are satisfied:

$$
\begin{aligned}
\phi_{M} \circ \phi_{M^{\prime}} & =\phi_{M^{\prime} M} \\
\left(\phi_{M}\right)^{-1} & =\phi_{M^{-1}} .
\end{aligned}
$$

Given a vector $\mu \in \mathbb{Z}^{n}$, we have

$$
\phi_{M}(x)^{\mu}=x^{\mu_{1} u^{(1)}} \cdots x^{\mu_{n} u^{(n)}}=x_{1}^{\sum_{i=1}^{n} \mu_{i} u_{1}^{(i)}} \cdots x_{n}^{\sum_{i=1}^{n} \mu_{i} u_{n}^{(i)}}=x^{M \mu} .
$$

We will denote by $L_{M}$, the linear map given by

$$
\begin{aligned}
L_{M}: \mathbb{R}^{n} & \longrightarrow \mathbb{R}^{n} \\
x & \longmapsto M x .
\end{aligned}
$$

Let $f$ be a polynomial in $n$ variables as in (2), equation (6) implies

$$
f \circ \phi_{M}(x)=\sum_{\mu \in \varepsilon(f)} a_{\mu} x^{M \mu}
$$


then

$$
\varepsilon\left(f \circ \phi_{M}\right)=M \cdot \varepsilon(f)=L_{M}(\varepsilon(f)) .
$$

Let $\pi$ be a supporting hyperplane for $N P(f)$, then $L_{M}(\pi)$ is a supporting hyperplane for $N P\left(f \circ \phi_{M}\right)$, and

$$
\left.f \circ \phi_{M}\right|_{L_{M}(\pi) \cap N P\left(f \circ \phi_{M}\right)}=\left.f\right|_{\pi \cap N P(f)} \circ \phi_{M} .
$$

Lemma 5.1. Let $M$ be an unimodular matrix with integer entries, we have

$$
L_{M}\left(\left(\operatorname{cone}\left(M^{t}\right)\right)^{\sqrt{ }}\right)=\left(\mathbb{R}_{\geq 0}\right)^{n} .
$$

Proof.

The dual cone $\left(M^{t}\right)^{\vee}$ is generated by $\left\langle v^{(1)}, \ldots, v^{(n)}\right\rangle$, where by $11, v^{(1)}, \ldots, v^{(n)}$ are the columns of the matrix $M^{-1}$.

$$
\begin{aligned}
L_{M}\left(\left(\operatorname{cone}\left(M^{t}\right)\right)^{\vee}\right) & =\left\{L_{M}\left(t_{1} v^{(1)}+\cdots+t_{n} v^{(n)}\right) ; t_{i} \in \mathbb{R}_{\geq 0}\right\} \\
& \left\{t_{1}\left(M v^{(1)}\right)+\cdots+t_{n}\left(M v^{(n)}\right) ; t_{i} \in \mathbb{R}_{\geq 0}\right\} \\
& =\left\{t_{1}(1,0, \ldots, 0)+\cdots+t_{n}(0, \ldots, 0,1) ; t_{i} \in \mathbb{R}_{\geq 0}\right\} \\
& =\left\{\left(t_{1}, \ldots, t_{n}\right) ; t_{i} \in \mathbb{R}_{\geq 0}\right\}=\left(\mathbb{R}_{\geq 0}\right)^{n}
\end{aligned}
$$

Hence we have the required result.

Proposition 5.2. Let $M \in G L(n, \mathbb{Z})$ be such that $\sigma=\operatorname{cone}\left(M^{t}\right)$ is $\operatorname{good}$ for $f$, then $N P\left(f \circ \phi_{M}\right)$ has only one vertex (i. e. $\mathbb{R}_{\geq 0}^{n}$ is good for $\left.f \circ \phi_{M}\right)$.

Proof.

Suppose that $\sigma=\operatorname{cone}\left(M^{t}\right)$ is good for $f$, then, by Remark 4.5 .

$$
\varepsilon(f) \subset \mathbf{P}+\sigma^{\sqrt{ }}=\mathbf{P}+\left(\operatorname{cone}\left(M^{t}\right)\right)^{\sqrt{ }}
$$

with $\mathbf{P} \in \varepsilon(f)$.

By (8) and (10),

$$
\varepsilon\left(f \circ \phi_{M}\right) \subset L_{M}(\mathbf{P})+L_{M}\left(\left(\operatorname{cone}\left(M^{t}\right)\right)^{\vee}\right)=L_{M}(\mathbf{P})+\left(\mathbb{R}_{\geq 0}\right)^{n}
$$

where the equality follows from Lemma 5.1 .

Now, since $\mathbf{P}$ is in the support of $f$, the point $L_{M}(\mathbf{P})$ is in the support of $f \circ \phi_{M}$. Then, $L_{M}(\mathbf{P})$ is the only vertex of $N P\left(f \circ \phi_{M}\right)$, and we have the result.

Proposition 5.3. Let $M \in G L(n, \mathbb{Z})$ be such that $\sigma=$ Cone $(M)$ is good for $f$. Given $\lambda \subset\{1, \ldots, n\}$, let $\tau$ be the face of $\sigma$ generated by the $i^{\text {th }}$ columns of $M$, with $i \in \lambda$. We have

$$
\left.f\right|_{\text {face }_{\tau}(f)} \circ \phi_{M^{t}}=\left.f \circ \phi_{M^{t}}\right|_{\mathbf{P}+\operatorname{Cone}\left(\left\{e^{(i)}\right\}_{i \in \lambda}\right)}
$$

where $\mathbf{P}$ is the only vertex of $N P\left(f \circ \phi_{M^{t}}\right)$.

Proof.

Consequence of equation (9) and Proposition 5.2. 


\section{Toric modification.}

In this section we will recall how to construct the modification associated to a regular fan $\Sigma$ with support in the first orthant. See for example [Oka2, Cap.2].

Let $\mathfrak{M}$ be the set of cones in $\Sigma$ of maximal dimension. Let $\sigma \in \Sigma$ be a cone in $\mathfrak{M}$. We will associate to $\sigma$ one copy of the affine space $\mathbb{K}^{n}$ and we will denote it by $U_{\sigma}$. Consider the disjoint union

$$
\mathfrak{C}=\bigsqcup_{\sigma \in \mathfrak{M}} U_{\sigma}
$$

Let $\sigma=\operatorname{Cone}(M)$ and $\sigma^{\prime}=$ Cone $\left(M^{\prime}\right)$ be cones in $\mathfrak{M}$.

Consider

$$
\phi_{\left(M^{-1}\right)^{t}}:\left(\mathbb{K}^{*}\right)^{n} \longrightarrow U_{\sigma} \quad \text { and } \quad \phi_{\left(M^{\prime-1}\right)^{t}}:\left(\mathbb{K}^{*}\right)^{n} \longrightarrow U_{\sigma^{\prime}} .
$$

The composition $\phi_{\left(M^{\prime-1}\right)^{t}} \circ \phi_{\left(M^{-1}\right)^{t}}^{-1}=\phi_{M^{t} .\left(M^{\prime-1}\right)^{t}}: U_{\sigma} \rightarrow U_{\sigma^{\prime}}$ is a birational morphism.

An equivalence relation is defined in $\mathfrak{C}$ as follows: Given two points $u_{\sigma} \in U_{\sigma}$ and $u_{\sigma^{\prime}}^{\prime} \in U_{\sigma^{\prime}}, u_{\sigma} \sim u_{\sigma^{\prime}}^{\prime}$ if and only if $\phi_{M^{t}\left(M^{\prime-1}\right)^{t}}$ is bi-regular on $u_{\sigma}$ and $\phi_{M^{t} .\left(M^{\prime-1}\right)^{t}}\left(u_{\sigma}\right)=u_{\sigma^{\prime}}^{\prime}$.

The quotient of $\mathfrak{C}$ under this equivalence relation is a smooth variety, $X_{\Sigma}$, called the toric variety associated to $\Sigma$. Each $U_{\sigma}$ is a chart of $X_{\Sigma}$.

For each $\sigma=\operatorname{Cone}(M) \in \mathfrak{M}$ the mapping

$$
\begin{aligned}
\left.\pi\right|_{U_{\sigma}}: U_{\sigma} & \longrightarrow \mathbb{K}^{n} \\
x & \mapsto \phi_{M^{t}}(x)
\end{aligned}
$$

is a regular morphism, compatible with the gluing.

The induced regular morphism

$$
\pi: X_{\Sigma} \longrightarrow \mathbb{K}^{n}
$$

is called the toric modification associated to $\Sigma$. The morphism $\pi$ is a proper bi-rational morphism and it is bi-regular in the torus $\left(\mathbb{K}^{*}\right)^{n}$.

Given a variety $V \subset \mathbb{K}^{n}$, the inverse image $\pi^{-1}(V)$ is called the total transform of $V$ under the projection $\pi$.

Let $V^{*} \subset V$ be the set of points in $V$ lying outside the coordinate hyperplanes, that is,

$$
V^{*}:=V-V\left(x_{1} \cdots x_{n}\right) .
$$

The strict transform of $V$ under the projection $\pi$ is defined by

$$
\widetilde{V}:=\overline{\pi^{-1}\left(V^{*}\right)}
$$

where $\bar{A}$ denotes the Zariski closure of $A$.

Given $\sigma \in \mathfrak{M}$, there is a natural action

$$
\begin{array}{ccc}
\left(\mathbb{K}^{*}\right)^{n} \times U_{\sigma} & \longrightarrow & U_{\sigma} \\
\left(\lambda_{1}, \ldots, \lambda_{n}\right) \times\left(z_{1}, \ldots, z_{n}\right) & \mapsto & \left(\lambda_{1} z_{1}, \ldots, \lambda_{n} z_{n}\right)
\end{array}
$$


that is compatible with the gluing in $X_{\Sigma}$.

Given $\lambda \subset\{1, \ldots, n\}$ and $\sigma=\operatorname{Cone}(M) \in \mathfrak{M}$, let $\tau$ be the cone generated by the $i^{\text {th }}$ columns of $M$ with $i \in \lambda$.

The orbit associated to $\tau$ is the $(n-\# \lambda)$-dimensional torus given by

$$
\mathcal{O}(\tau):=\left\{\left(x_{1}, \ldots, x_{n}\right) ; x_{i}=0 \text { for } i \in \lambda \text { and } x_{i} \neq 0 \text { for } i \notin \lambda\right\} .
$$

The set $\mathcal{O}(\tau)$ is well defined (it does not depend on the choice of $\sigma$ ) and is an orbit of the natural action on $X_{\Sigma}$.

Remark 6.1. Let $\sigma=$ Cone $(M) \in \Sigma$ be of maximal dimension. Let $\tau$ be the cone in $\Sigma$ generated by the first $s$ columns of $M$. And let $h: U_{\sigma} \cap X_{\Sigma} \longrightarrow \mathbb{K}$ be a regular function.

By Remark 3.4, we have

$$
\left.h\right|_{\mathcal{O}(\tau) \cap U_{\sigma}}=\left.h\right|_{\operatorname{Cone}\left(e^{(s+1)}, \ldots, e^{(n)}\right)} .
$$

\section{Newton non degenerate hypersurfaces.}

A hypersurface singularity $\underline{0} \in H=V(f)$ is said to be Newton non degenerate when, for any face $F$ of $N P(f)$, the hypersurface $V\left(\left.f\right|_{F}\right)$ has no singularities outside the coordinate hyperplanes. In this section we will prove that a Newton non degenerate hypersurface singularity has a toric resolution. This result is proved in several texts (see for example [Tei, Mer).

Let $\Sigma$ be a regular refinement of $\Sigma(f)$, let $\{\underline{0}\} \neq \tau$ be a cone in $\Sigma$, and let $y$ be a point in the strict transform of $H$ under the toric modification given by $\Sigma$ with

$$
y \in \widetilde{H} \cap \mathcal{O}(\tau) \subset X_{\Sigma} .
$$

Choose $\sigma=\operatorname{Cone}(M) \in \mathfrak{M}$ such that the first $s$ columns of $M$ generate $\tau$. We have

$$
y=\left(0, \ldots, 0, y_{s+1}, \ldots, y_{n}\right) \in U_{\sigma}
$$

with $y_{i} \neq 0$ for $i \geq s+1$.

Since $\operatorname{Cone}(M)$ is good for $f$, by Proposition 5.2, $N P\left(f \circ \phi_{M^{t}}\right)$ has only one vertex P. By Remark 3.3, we have

$$
f \circ \phi_{M^{t}}=x^{\mathbf{P}} h
$$

with $h(\underline{0}) \neq 0$.

Then

$$
\widetilde{H} \cap U_{\sigma}=V(h)
$$

and, since $\underline{0} \notin V(h), \tau \neq \sigma$.

By Remark 6.1

$$
\left.h\right|_{\mathcal{O}(\tau) \cap U_{\sigma}}=\left.h\right|_{\text {Cone }\left(e^{(s+1)}, \ldots, e^{(n)}\right)},
$$

thus, the function $\left.h\right|_{\text {Cone }\left(e^{(s+1)}, \ldots, e^{(n)}\right)}$ does not depend on the first $s$ variables, then

$$
h(y)=\left.h\right|_{\text {Cone }\left(e^{(s+1)}, \ldots, e^{(n)}\right)}\left(1, \ldots, 1, y_{s+1}, \ldots, y_{n}\right) .
$$


By Proposition 5.3 .

$$
\left.x^{\mathbf{P}} h\right|_{\text {Cone }\left(e^{(s+1)}, \ldots, e^{(n)}\right)}=\left.f \circ \phi_{M^{t}}\right|_{\mathbf{P}+\operatorname{Cone}\left(e^{(s+1)}, \ldots, e^{(n)}\right)}=\left.f\right|_{\text {face }_{\tau}(f)} \circ \phi_{M^{t}}
$$

The hypersurface, $V\left(\left.f\right|_{\text {face }_{\tau}(f)}\right)$ has no singularities outside the coordinate hyperplanes, then, $V\left(\left.h\right|_{\text {Cone }\left(e^{(s+1)}, \ldots, e^{(n)}\right)}\right)$ is non singular in $\left(1, \ldots, 1, y_{s+1}, \ldots, y_{n}\right)$. That is, there exists $j \in\{1, \ldots, n\}$ such that

$$
\frac{\left.\partial h\right|_{\text {Cone }\left(e^{(s+1)}, \ldots, e^{(n)}\right)}}{\partial x_{j}}\left(1, \ldots, 1, y_{s+1}, \ldots, y_{n}\right) \neq 0
$$

since $\left.h\right|_{\text {Cone }\left(e^{(s+1)}, \ldots, e^{(n)}\right)}$ does not depend on the first $s$ coordinates, we have $j \geq s+1$.

By equation 12

$\frac{\partial h}{\partial x_{j}}(y)=\frac{\left.\partial h\right|_{\text {Cone }\left(e^{(s+1)}, \ldots, e^{(n)}\right)}}{\partial x_{j}}(y)=\frac{\left.\partial h\right|_{\text {Cone }\left(e^{(s+1)}, \ldots, e^{(n)}\right)}}{\partial x_{j}}\left(1, \ldots, 1, y_{s+1}, \ldots y_{n}\right) \neq 0$

that is, $y$ is not a singular point of $\widetilde{H}$ and is transversal to $\mathcal{O}(\tau)$.

\section{The Gröbner fan.}

The Gröbner fan is the extension of the concept of dual fan to non principal ideals. All this may be computed using the program GFAN [Jen].

Given $\omega \in\left(\mathbb{R}_{\geq 0}\right)^{n}$, the $\omega$-initial part of $f$ is

$$
\operatorname{In} f:=\left.f\right|_{\text {face }_{\omega}(f)} .
$$

That is, given $f$ as in $(2)$,

$$
\operatorname{In}_{\omega} f=\sum_{\left\{\mu \in \varepsilon(f) ; \omega \cdot \mu=\nu_{\omega} f\right\}} a_{\mu} x^{\mu} .
$$

Remark 8.1. Given $f \in \mathbb{K}\left[x_{1}, \ldots, x_{n}\right]$, if $f(\underline{0}) \neq 0$ then $\nu_{\omega}(u)=0$ for all $\omega \in\left(\mathbb{R}_{\geq 0}\right)^{n}$. And $\operatorname{In} \omega f=f(\underline{0})$ for all $\omega \in\left(\mathbb{R}_{>0}\right)^{n}$.

Let $I \subset \mathbb{K}\left[x_{1}, \ldots, x_{n}\right]$ be an ideal. Given $\omega \in\left(\mathbb{R}_{\geq 0}\right)^{n}$, the $\omega$-initial ideal of $I$ is the ideal generated by the $\omega$-initial part of every polynomial in $I$, that is,

$$
\mathfrak{I n}_{\omega} I:=\left\langle\operatorname{In}_{\omega} f ; f \in I\right\rangle .
$$

The variety $V\left(\mathfrak{I n}_{(1, \ldots, 1)} I\right)$ is classically called the tangent cone of $V(I)$.

In the light of remark 4.2 , there is a natural way to associate a polyhedral fan to an ideal. In $\mathbb{R}^{n}$, we define an equivalence relation as follows:

$$
\omega \sim \omega^{\prime} \Leftrightarrow \mathfrak{I n}_{\omega} I=\mathfrak{I n}_{\omega^{\prime}} I .
$$


Given $\omega \in \mathbb{R}^{n}$, the closure in $\mathbb{R}^{n}$ of its equivalence class, that is,

$$
C_{\omega}(I):=\overline{\left\{u \in \mathbb{R}^{n} ; \mathfrak{I n}_{u} I=\mathfrak{I n}_{\omega} I\right\}}
$$

is a polyhedral cone and the collection

$$
\Sigma^{\prime}(I):=\left\{C_{\omega}(I) ; \omega \in \mathbb{R}^{n}\right\}
$$

forms a fan ( MoRo $)$.

Since we are interested in a local study of a variety, we will use the fan formed by the intersection of this fan and the first orthant, that is,

$$
\Sigma(I)=\left\{\Sigma^{\prime}(I) \bigcap E_{J} ; J \subset\{1,2, \ldots, n\}\right\} \subset\left(\mathbb{R}_{\geq 0}\right)^{n}
$$

where $E_{J}=\operatorname{Cone}\left(\left\{e^{(j)} ; j \in J\right\}\right)$ and $e^{(j)}$ is the $j$-th element of the standard basis for $\mathbb{R}^{n}$. We will refer to $\Sigma(I)$ as the Gröbner fan of the ideal $I$. The cones in the Gröbner fan of $I$ will be called Gröbner cones of $I$.

Given $f \in \mathbb{K}\left[x_{1}, \ldots, x_{n}\right]$, we have

$$
\Sigma(f)=\Sigma(\langle f\rangle) .
$$

Remark 8.2. Given $\omega, \omega^{\prime} \in I n t_{r e l}\left\langle e^{\left(i_{1}\right)}, \ldots, e^{\left(i_{s}\right)}\right\rangle$ we have that the initial ideals $\mathfrak{I n}_{\omega} I$ and $\mathfrak{I n}_{\omega^{\prime}} I$ are equal if and only if $C_{\omega}(I)=C_{\omega^{\prime}}(I)$.

Remark 8.3. Let $\sigma$ be a cone in $\Sigma(I)$ not contained in the coordinate hyperplanes. The cone $\sigma$ is of maximal dimension if and only if $\mathfrak{I n}_{\sigma} I$ is a monomial ideal.

Definition 8.4. We will say that $\sigma$ is a good cone for $I$ when it is contained in a Gröbner cone of $I$.

Let $I \subseteq \mathbb{K}\left[x_{1}, x_{2}, \ldots, x_{n}\right]$ be an ideal and let $\sigma$ be a good cone for $I$. We define

$$
\mathfrak{I n}_{\sigma} I:=\mathfrak{I n}_{\omega} I \quad \text { where } \quad \omega \in \operatorname{Int}_{\text {rel }}(\sigma) .
$$

Remark 8.5. Let $I \subseteq \mathbb{K}\left[x_{1}, x_{2}, \ldots, x_{n}\right]$ be an ideal, let $\sigma$ be a good cone for $I$ and let $\tau$ be a face of $\sigma$. Then, $\tau$ is a good cone for $I$ and

$$
\mathfrak{I n}_{\sigma} \mathfrak{I n}_{\tau} I=\mathfrak{I n}_{\sigma} I .
$$

\section{Reduced Gröbner basis}

Given a term order, $\prec$, we define the initial term, $I n_{\prec} f$, of a non-zero polynomial $f \in \mathbb{K}\left[x_{1}, \ldots, x_{n}\right]$, as the unique minimal term with respect to $\prec$.

Let $I \subset \mathbb{K}\left[x_{1}, \ldots, x_{n}\right]$ be an ideal. The initial ideal of $I$ with respect to $\prec$ is the ideal generated by the initial terms of the polynomials in $I$, that is,

$$
\mathfrak{I n}_{\prec} I:=\left\langle I n_{\prec} f ; f \in I\right\rangle .
$$

The closure of the equivalence class:

$$
C_{\prec}(I):=\overline{\left\{u \in \mathbb{R}^{n} ; \mathfrak{I n}_{u} I=\mathfrak{I n}_{\prec} I\right\}}
$$

is a Gröbner cone of maximal dimension. 
Proposition 9.1. Let $I \subset \mathbb{K}\left[x_{1}, \ldots, x_{n}\right]$ be an ideal and let $\prec$ be a term order. There exist a set $G_{\prec}(I)=\left\{g_{1}, \ldots, g_{r}\right\} \subset I$ (called reduced Gröbner basis) such that

i) $C_{\prec}(I)$ is a good cone for $g_{i}, i \in\{1, \ldots, r\}$.

ii) $\mathfrak{I n}_{v}(I)=\left\langle\operatorname{In}_{v}\left(g_{1}\right), \ldots, \operatorname{In} n_{v}\left(g_{r}\right)\right\rangle$, for all $v \in C_{\prec}(I)$.

Proof.

i) Direct consequence of [Prop 2.6, [FJT]

ii) Direct consequence of [Coro 2.14, [FJT]].

Given $\omega \in\left(\mathbb{R}_{\geq 0}\right)^{n}$, we define a total order $\prec_{\omega}$ in $\mathbb{K}\left[x_{1}, \ldots, x_{n}\right]$ by,

$$
x^{\alpha} \prec_{\omega} x^{\beta} \Leftrightarrow \alpha \cdot \omega<\omega \cdot \beta \text { or } \alpha \cdot \omega=\omega \cdot \beta \text { and } \alpha \prec_{\text {lex }} \beta,
$$

where $\prec_{l e x}$ is the lexicographical order.

Proposition 9.2. Let $I \subset \mathbb{K}\left[x_{1}, \ldots, x_{n}\right]$ be an ideal. Given $\sigma \in \Sigma(I)$ with $\operatorname{dim}(\sigma)=n$ and $\omega \in \operatorname{Int}_{\text {rel }}(\sigma)$ we have that $\sigma=C_{\prec \omega}(I)$.

Proof. Take $\omega \in \operatorname{Int}_{r e l}(\sigma)$ and let $\prec_{\omega}$ be as in (15), then, by definition, we have $\mathfrak{I n}_{C_{\prec_{\omega}}}(I)=\mathfrak{I n}_{\prec_{\omega}}(I)$ and $\mathfrak{I n}_{\omega}(I)=\mathfrak{I n}_{\sigma}(I)$. Also, $\mathfrak{I n}_{\prec_{\omega}}(I)=\mathfrak{I n}_{\prec_{\omega}} \mathfrak{I n}_{\omega}(I)$ (by [Lemma 2.13, [FJT] ]) and $\mathfrak{I n}_{\prec \omega} \mathfrak{I n}_{\omega}(I)=\mathfrak{I n}_{\omega}(I)$ (by Remark 8.3).

All above equations imply $\mathfrak{I n}_{C_{\prec}}(I)=\mathfrak{I n}_{\sigma}(I)$.

Proposition 9.3. Let $I \subset \mathbb{K}\left[x_{1}, \ldots, x_{n}\right]$ be an ideal and let $\sigma$ be a good cone for $I$ of maximal dimension. There exists a system of generators $G_{\sigma}=\left\{g_{1}, \ldots, g_{r}\right\}$ of $I$ such that $\sigma$ is a good cone for $g_{i}$ for $i \in\{1, \ldots, r\}$ and

$$
\mathfrak{I n}_{v}(I)=\left\langle\operatorname{In}_{v} g_{1}, \ldots, \operatorname{In}_{v} g_{r}\right\rangle
$$

for all $v \in \sigma$.

Proof. Let $\sigma^{\prime} \in \Sigma(I)$ be such that $\sigma \subset \sigma^{\prime}$ (exists by definition of good cone). Take $\omega \in I n t_{r e l} \sigma^{\prime}$ and consider the term order $\prec_{\omega}$. By Proposition 9.2 we have

$$
\sigma \subset \sigma^{\prime}=C_{\prec \omega}(I) .
$$

By Prop 9.1, there exists a system of generators $g_{1}, \ldots, g_{r}$ of $I$, such that $C_{\prec_{\omega}}(I)$ is good for each $g_{i}$. The result follows from 16 . 


\section{Tropical Variety}

Definition 10.1. Given an ideal $I \subset \mathbb{K}\left[x_{1}, \ldots, x_{n}\right]$, the tropical variety associated to $I$ is

$$
\mathbf{T V}(I):=\left\{\omega \in \mathbb{R}^{n} ; \mathfrak{I n}_{\omega}(I) \text { contains no monomial }\right\} .
$$

Proposition 10.2. An ideal $I \subset \mathbb{K}\left[x_{1}, \ldots, x_{n}\right]$ contains no monomials if and only if $V(I) \cap\left(\mathbb{K}^{*}\right)^{n} \neq \emptyset$.

Proof. $\Leftarrow]$ If $a x^{\alpha} \in I$ and $z \in V(I)$, then $a z_{1}^{\alpha_{1}} \cdots z_{n}^{\alpha_{n}}=0$ so there exist $i$ such that $z_{i}=0$. Hence, $z \notin\left(\mathbb{K}^{*}\right)^{n}$.

$\Rightarrow$ ] Suppose that $V(I) \cap\left(\mathbb{K}^{*}\right)^{n}=\emptyset$. Then $V(I) \subset \bigcup_{i=1}^{n}\left\{x_{i}=0\right\}$. The monomial $x^{(1, \ldots, 1)}$ satisfies $V\left(x^{(1, \ldots, 1)}\right) \supset V(I)$ and, by the Nullstellensatz, there exists $k$ such that $x^{(k, \ldots, k)} \in I$, i.e. $I$ contains a monomial.

Proposition 10.2 implies

$$
\mathbf{T V}(I)=\left\{\omega \in \mathbb{R}^{n} ; V\left(\mathfrak{I n}_{\omega}(I)\right) \cap\left(\mathbb{K}^{*}\right)^{n} \neq \emptyset\right\} .
$$

Proposition 10.3. Let $I \subset \mathbb{K}\left[x_{1}, \ldots, x_{n}\right]$ be an ideal. Given $\omega \in \operatorname{Int}_{r e l}(\sigma)$ with $\sigma \in \Sigma(I)$ a cone. We have

$$
\omega \in \mathbf{T V}(I) \Longleftrightarrow \sigma \subset \mathbf{T V}(I) .
$$

Proof.

$\Leftarrow$ Suppose that $\sigma$ is contained in $\mathbf{T V}(I)$ then $\omega \in \operatorname{Int}_{\text {rel }}(\sigma) \subset \sigma$, implies $\omega \in \mathbf{T V}(I)$.

$\Rightarrow$ Let $\sigma \subset \Sigma(I)$ be a cone. Let $\omega \in \operatorname{Int}_{r e l}(\sigma)$ be such that $\omega \in \mathbf{T V}(I)$.

- Take $\omega^{\prime} \in \operatorname{Int}_{r e l}(\sigma)$, by Remark $8.2 \omega^{\prime} \in \mathbf{T V}(I)$.

- Take $\nu \in \sigma \backslash \operatorname{Int}_{r e l}(\sigma)$. There exist $\tau \in \Sigma(I)$ face of $\sigma$ such that $\nu \in \operatorname{Int}_{\text {rel }}(\tau)$. By duality we have

$$
\varepsilon\left(\operatorname{In}_{\omega}(f)\right)=\varepsilon\left(\operatorname{In}_{\sigma}(f)\right) \subset \varepsilon\left(\operatorname{In}_{\tau}(f)\right)=\varepsilon\left(\operatorname{In}_{\nu}(f)\right) .
$$

Then, if $I n_{\omega} f$ is not a monomial neither is $I n_{\nu} f$.

From the last proposition, it follows that the tropical variety is a union of cones in the Gröbner fan.

Theorem 10.4. Bieri-Groves $[B i G r,[S t u]$ Let $\mathbb{K}$ be algebraically closed field and let $I \subset \mathbb{K}\left[x_{1}, \ldots, x_{n}\right]$ be a monomial-free prime ideal. Then the tropical variety $\mathbf{T V}(I)$ can be written as a finite union of polyhedra of dimension dim $V(I)$.

Proposition 10.5. Let $\mathbb{K}$ be an algebraically closed field and let $V=V(I) \subset \mathbb{K}^{n}$ be a pure dimensional variety not contained in the coordinate hyperplanes. Given $\omega \in \mathbf{T V}(I)$, we have

$$
\operatorname{dim} \mathbf{T V}(I)=\operatorname{dim} \mathbf{T V}\left(\mathfrak{I n}_{\omega} I\right)
$$


Proof. Suppose that $\nu \notin \mathbf{T V}(I)$ then, there exists $f \in I$ such that $\operatorname{In}_{\nu} f$ is a monomial and, then, $I n_{\nu} I n_{\omega} f$ is also a monomial. Since $I n_{\omega} f$ is in $\mathfrak{I n}_{\omega} I$ we have $\nu \notin \mathbf{T V}\left(\mathfrak{I n}_{\omega} I\right)$. This gives the inclusion

$$
\mathbf{T V}\left(\mathfrak{I n}_{\omega} I\right) \subset \mathbf{T V}(I) .
$$

Now, let $d$ be the dimension of $\mathbf{T V}(I)$. Given $\omega$ in the tropical variety $\mathbf{T V}(I)$, by Theorem 10.4 there exists a cone $\sigma$ that is a good cone for $I$ with $\omega \in \sigma$ and $\operatorname{dim} \sigma=d$. By Remark 8.5, $\mathfrak{I n}_{\sigma} \mathfrak{I n}_{\omega} I=\mathfrak{I n}_{\sigma} I$. Since $\mathfrak{I n}_{\sigma} I$ is not a monomial we have $\sigma \subset \mathbf{T V}\left(\mathfrak{I n}_{\omega} I\right)$.

Then

$$
\operatorname{dim} \mathbf{T V}\left(\mathfrak{I n}_{\omega} I\right) \geq \mathbf{T V}(I) .
$$

And the result is prooved.

Proposition 10.6. Let $\mathbb{K}$ be an algebraically closed field and let $V=V(I) \subset \mathbb{K}^{n}$ be a pure dimensional variety not contained in the coordinate hyperplanes. Given $\omega \in \mathbf{T V}(I)$, we have

$$
\operatorname{dim}\left(V \cap \mathbb{K}^{* n}\right)=\operatorname{dim} \mathbf{T V}(I)=\operatorname{dim} \mathbf{T V}\left(\mathfrak{I n}_{\omega} I\right) .
$$

Proof. Direct consequence of Theorem 10.4 and Proposition 10.5 .

\section{Newton non-degenerate varieties.}

In this section we will extend the concept of Newton non degenerate variety to non-principal ideals. For complete intersections the concept was extended by Khovanskii [Kho2, Kho1] in 1976.

Definition 11.1. Let $V=V\left(f_{1}, \ldots, f_{k}\right) \subset \mathbb{K}^{n}$ be a variety of dimension $n-k$. The variety $V$ is Newton non-degenerate if for any $\omega \in\left(\mathbb{R}_{\geq 0}\right)^{n}$ the variety

$$
V\left(\operatorname{In}_{\omega} f_{1}, \operatorname{In}_{\omega} f_{2}, \ldots, \operatorname{In} n_{\omega} f_{k}\right)
$$

is of dimension $n-k$ and has no singularities in $\left(\mathbb{K}^{*}\right)^{n}$.

Let $I$ be the ideal generated by $f_{1}, \ldots, f_{k}$, then, $V\left(f_{1}, \ldots, f_{k}\right)=V(I)$. Given an other set of generators of $I$, say, $f_{1}^{\prime}, \ldots, f_{k}^{\prime}$. The ideals

$$
\left\langle\operatorname{In}_{\omega} f_{1}, \operatorname{In} n_{\omega} f_{2}, \ldots, I n_{\omega} f_{k}\right\rangle \text { and }\left\langle\operatorname{In} n_{\omega} f_{1}^{\prime}, \operatorname{In} n_{\omega} f_{2}^{\prime}, \ldots, I n_{\omega} f_{k}^{\prime}\right\rangle
$$

are not necessarily the same. Definition 11.1 depends strongly on the generators of the ideal $I$ chosen.

The definition we propose extends the definition above to non complete intersection singularities and does not depend on the generators.

Definition 11.2. A singularity $\underline{0} \in V(I) \subset \mathbb{K}^{n}$, where $I \subset \mathbb{K}\left[x_{1}, \ldots, x_{n}\right]$ is an ideal, is Newton non-degenerate if for every $\omega \in\left(\mathbb{R}_{\geq 0}\right)^{n}$, the variety defined by $\mathfrak{I n}_{\omega} I$ does not have singularities in $\left(\mathbb{K}^{*}\right)^{n}$. 
To check if an ideal is Newton non degenerate or not, it is enough to check the condition for one vector in the relative interior of each cone of the Gröbner fan that is contained in the tropical variety associated to $I$. These computations may be done using the software GFAN [Jen].

Let $I=\left\langle f_{1}, f_{2}, f_{3}\right\rangle \subset \mathbb{C}[x, y, z, w]$ where

$$
f_{1}=x y+x w-y w, f_{2}=x z-w^{2} \text { and } f_{3}=y z-y w-w^{2} .
$$

The zero set $V(I)$ is a surface that has a rational singularity of multiplicity three (see [LeTo, or Tju).

In Gom the Groebner fan and the different initial ideals are computed using GFAN [Jen. Then, their singularities are computed using SINGULAR DGPS concluding that it is a Newton non degenerate singularity in the sense of Definition 11.2

Let $V=V\left(f_{1}, \ldots, f_{k}\right) \subset \mathbb{K}^{n}$ be Newton non degenerate variety in the sense of Definition 11.1. Let $\Sigma$ be a regular refinement of the dual fan $\Sigma\left(f_{i}\right)$ defined by $f_{i}$ for $i=1, \ldots, k$ and let $\pi: X_{\Sigma} \longrightarrow \mathbb{K}^{n}$ be the torical modification associated to $\Sigma$. Let $\widetilde{V}$ be the strict transform of $V$. It is well-known Oka2 that the restriction $\pi: \widetilde{V} \longrightarrow V$ is a good resolution of $V$. The following sections are devoted to show the corresponding result for Newton non degenerate varieties in the sense of our definition (Definition 11.2).

\section{Tropical variety and strict transform}

Let $I \subset \mathbb{K}\left[x_{1}, \ldots, x_{n}\right]$ be an ideal, let $\Sigma$ be a regular refinement of the Gröbner fan $\Sigma(I)$, and let $\widetilde{V(I)}$ be the strict transform of $V(I)$ under the modification

$$
\pi: X_{\Sigma} \longrightarrow \mathbb{K}^{n}
$$

Let $\sigma=\operatorname{Cone}(M) \in \Sigma$ be a cone of maximal dimension and let $G_{\sigma}$ be a system of generators as in Proposition 9.3

We have

$$
\phi_{M^{t}{ }_{*}}(I)=\left\langle\left\{g \circ \phi_{M^{t}}\right\}_{g \in G_{\sigma}}\right\rangle .
$$

Take $g \in G_{\sigma}$. Since Cone $(M)$ is good for $g$, by Proposition 5.2 and Remark 3.3 we have

$$
g \circ \phi_{M^{t}}=x^{\mathbf{P}} h
$$

with $h(\underline{0}) \neq 0$.

Set

$$
H_{G_{\sigma}}:=\left\{h \in \mathbb{K}\left[x_{1}, \ldots, x_{n}\right] ; g \circ \phi_{M^{t}}=x^{\mathbf{P}} h \text { for some } g \in G_{\sigma} \text { and } h(\underline{0}) \neq 0\right\} .
$$

We have

$$
\phi_{M^{t} *}(I) \subset\left\langle H_{G_{\sigma}}\right\rangle \quad \text { and } \quad V\left(\phi_{M^{t} *}(I)\right) \cap \mathbb{K}^{* n}=V\left(H_{G_{\sigma}}\right) \cap \mathbb{K}^{* n}
$$


then

$$
U_{\sigma} \cap \widetilde{V(I)} \subseteq V\left(H_{G_{\sigma}}\right) \stackrel{\sqrt[23]{\subseteq}}{\subseteq} U_{\sigma} \cap \pi^{-1}(V(I)) .
$$

In the following section we will see that, when $I$ is Newton non degenerate, $V\left(H_{G_{\sigma}}\right)$ is non singular and transversal to $\pi^{-1}(\underline{0})$. As a consequence, the first inclusion is actually an equality. ${ }^{1}$

Proposition 12.1. Let $I \subset \mathbb{K}\left[x_{1}, \ldots, x_{n}\right]$ be an ideal, let $\Sigma$ be a regular refinement of the Gröbner fan $\Sigma(I)$, and let $\widetilde{V(I)}$ be the strict transform of $V(I)$ under the modification given by $\Sigma$.

Given $\tau \in \Sigma$ we have

$$
\widetilde{V(I)} \cap \mathcal{O}(\tau) \neq \emptyset \Rightarrow \tau \subset \mathbf{T V}(I) .
$$

Proof. Given $\tau \in \Sigma$, take $\sigma=\operatorname{Cone}(M) \in \Sigma$, of maximal dimension, such that $\tau$ is generated by the first $s$ columns of $M$.

Take $y \in \widetilde{V(I)} \cap \mathcal{O}(\tau)$, we have

$$
y=\left(0, \ldots, 0, y_{s+1}, \ldots, y_{n}\right) \in U_{\sigma}, \quad \text { with } \quad y_{i} \neq 0 \quad \text { for } \quad i \in\{s+1, \ldots, n\} .
$$

Set

$$
y^{\prime}=\left(1, \ldots, 1, y_{s+1}, \ldots, y_{n}\right) \in U_{\sigma} \cap\left(\mathbb{K}^{*}\right)^{n} .
$$

Let $G_{\sigma}$ be a system of generators as in Proposition 9.3. Let $H_{G_{\sigma}}$ be as in 20]. Take $g \in G_{\sigma}$ and $h \in H_{G_{\sigma}}$ with $g \circ \phi_{M^{t}}=x^{\mathbf{P}} h$. By Remark 3.4. the function $\left.h\right|_{\text {Cone }\left(e^{(s+1)}, \ldots, e^{(n)}\right)}$ does not depend on the first $s$ variables, then

$$
\left.h\right|_{\text {Cone }\left(e^{(s+1)}, \ldots, e^{(n)}\right)}\left(y^{\prime}\right)=\left.h\right|_{\text {Cone }\left(e^{(s+1)}, \ldots, e^{(n)}\right)}(y)=h(y) \stackrel{211}{=} 0 .
$$

where the second inequality follows from Remark 6.1.

By Proposition 5.3 .

$$
\left.x^{\mathbf{P}} h\right|_{\text {Cone }\left(e^{(s+1)}, \ldots, e^{(n)}\right)}=\left.g \circ \phi_{M^{t}}\right|_{\mathbf{P}+\operatorname{Cone}\left(e^{(s+1)}, \ldots, e^{(n)}\right)}=\left.g\right|_{f a c e_{\tau}(g)} \circ \phi_{M^{t}}
$$

then

$$
\phi_{M^{t}}\left(y^{\prime}\right) \in V\left(\operatorname{In}_{\tau}(g)\right) \text { for all } g \in G_{\sigma} .
$$

By Proposition 9.3

$$
\mathfrak{I n}_{\tau}(I)=\left\langle\left\{I n_{\tau} g\right\}_{g \in G_{\sigma}}\right\rangle .
$$

then

$$
\phi_{M^{t}}\left(y^{\prime}\right) \in V\left(\mathfrak{I n}_{\tau}(I)\right) .
$$

Since $y^{\prime} \in\left(\mathbb{K}^{*}\right)^{n}$ then $\phi_{M^{t}}\left(y^{\prime}\right) \in\left(\mathbb{K}^{*}\right)^{n}$, and the implication follows from 17 .

\footnotetext{
${ }^{1}$ Since $G_{\sigma}$ is a Gröbner base, it is in particular a normalised standard base and the equality holds always (See for example [Hir or, for the analytic case, [AHV]
} 


\section{Toric resolution}

Theorem 13.1. Let $I \subset \mathbb{K}\left[x_{1}, \ldots, x_{n}\right]$ be an ideal and let $\Sigma$ be a regular refinement of the Gröbner fan of I. If $\underline{0} \in V=V(I)$ is a Newton non-degenerate singularity, then the strict transform $\widetilde{V}$ of $V$ under the toric modification

$$
\pi: X_{\Sigma} \longrightarrow \mathbb{K}^{n}
$$

associated to $\Sigma$ is non-singular.

Proof. Let $\Sigma$ be a regular refinement of $\Sigma(I)$, let $\{\underline{0}\} \neq \tau$ be a cone in $\Sigma$, and let $y$ be a point in the strict transform of $V(I)$ under the toric modification given by $\Sigma$ with

$$
\widetilde{V(I)} \cap \mathcal{O}(\tau) \subset X_{\Sigma}
$$

Choose $\sigma=$ Cone $(M)$ of maximal dimension such that the first $s$ columns of $M$ generate $\tau$. We have

$$
y=\left(0, \ldots, 0, y_{s+1}, \ldots, y_{n}\right) \in U_{\sigma}
$$

with $y_{i} \neq 0$ for $i \geq s+1$.

Set

$$
y^{\prime}=\left(1, \ldots, 1, y_{s+1}, \ldots, y_{n}\right) \in U_{\sigma} \cap\left(\mathbb{K}^{*}\right)^{n} .
$$

Let $G_{\sigma}=\left\{g_{1}, \ldots, g_{r}\right\}$ be a system of generators as in Proposition 9.3 The cone $\sigma$ is good for each $g \in G_{\sigma}$ and

$$
\mathfrak{I n}_{\tau}(I)=\left\langle\left\{I n_{\tau} g\right\}_{g \in G_{\sigma}}\right\rangle .
$$

The set $G_{\sigma}$ is a system of generators of $I$, then

$$
\phi_{M^{t} *}(I)=\left\langle\left\{g \circ \phi_{M^{t}}\right\}_{g \in G_{\sigma}}\right\rangle
$$

Let $H_{G_{\sigma}}=\left\{h_{1}, \ldots, h_{r}\right\}$ be as in 20$)$. We have

$$
\widetilde{V} \cap U_{\sigma} \subseteq V\left(h_{1}, \ldots, h_{r}\right) .
$$

By Remark 6.1.

$$
\left.h_{i}\right|_{\mathcal{O}(\tau) \cap U_{\sigma}}=\left.h_{i}\right|_{\text {Cone }\left(e^{(s+1)}, \ldots, e^{(n)}\right)} .
$$

The functions $\left.h_{i}\right|_{\text {Cone }\left(e^{(s+1)}, \ldots, e^{(n)}\right)}$ do not depend on the first $s$ variables, hence

$$
h_{i}(y)=\left.h_{i}\right|_{\operatorname{Cone}\left(e^{(s+1)}, \ldots, e^{(n)}\right)}\left(y^{\prime}\right) .
$$

By Proposition 5.3

$$
\left.x^{\mathbf{P}_{i}} h_{i}\right|_{\text {Cone }\left(e^{(s+1)}, \ldots, e^{(n)}\right)}=\left.g_{i} \circ \phi_{M^{t}}\right|_{\mathbf{P}_{i}+\operatorname{Cone}\left(e^{(s+1)}, \ldots, e^{(n)}\right)}=I n_{\tau} g_{i} \circ \phi_{M^{t}} .
$$


Continuing as in the proof of Proposition $12.1 \tau \subset \mathbf{T V}(I)$. Then, by Proposition 10.6

$$
\begin{aligned}
\operatorname{dim} V & =\operatorname{dim} \mathbf{T V}(V)=\operatorname{dim} \mathbf{T V}\left(\mathfrak{I n}_{\tau}(I)\right) \\
& \stackrel{[22}{=} \operatorname{dim} V\left(\operatorname{In}_{\tau} g_{1}, \ldots, \operatorname{In}_{\tau} g_{r}\right) \\
& =\operatorname{dim} V\left(\left.h_{1}\right|_{\text {Cone }\left(e^{(s+1)}, \ldots, e^{(n)}\right)}, \ldots,\left.h_{r}\right|_{\text {Cone }\left(e^{(s+1)}, \ldots, e^{(n)}\right)}\right)
\end{aligned}
$$

The variety $V\left(I n_{\tau} g_{1}, \ldots, I n_{\tau} g_{r}\right)$ has no singularities outside the coordinate hyperplanes, then, $V\left(\left.h_{1}\right|_{\text {Cone }\left(e^{(s+1)}, \ldots, e^{(n)}\right)}, \ldots,\left.h_{r}\right|_{\text {Cone }\left(e^{(s+1)}, \ldots, e^{(n)}\right)}\right)$ is non singular in $y^{\prime}$. That is,

$$
\operatorname{Rank}\left(\frac{\left.\partial h_{j}\right|_{\operatorname{Cone}\left(e^{(s+1)}, \ldots, e^{(n)}\right)}}{\partial x_{i}}\right)\left(y^{\prime}\right)=\operatorname{dim} V
$$

Since $\left.h_{j}\right|_{\text {Cone }\left(e^{(s+1)}, \ldots, e^{(n)}\right)}$ does not depend on the first $s$ variables

$$
\operatorname{Rank}\left(\frac{\left.\partial h_{j}\right|_{\text {Cone }\left(e^{(s+1)}, \ldots, e^{(n)}\right)}}{\partial x_{i}}\right)_{j=1, \ldots, r, i=s+1, \ldots, n}\left(y^{\prime}\right)=\operatorname{dim} V
$$

Now

$$
\begin{gathered}
\left(\frac{\left.\partial h_{j}\right|_{\text {Cone }\left(e^{(s+1)}, \ldots, e^{(n)}\right)}}{\partial x_{i}}\right)_{j=1, \ldots, r, i=s+1, \ldots, n}\left(y^{\prime}\right) \\
=\left(\frac{\left.\left.\partial h_{j}\right|_{\text {Cone }(e(s+1), \ldots, e(n)}\right)}{\partial x_{i}}\right)_{j=1, \ldots, r, i=s+1, \ldots, n}(y) \\
=\left(\frac{\partial h_{j}}{\partial x_{i}}\right)_{j=1, \ldots, r, i=s+1, \ldots, n}(y) .
\end{gathered}
$$

Then $V\left(H_{G_{\sigma}}\right)$ is non singular and transversal to $\pi^{-1}(\underline{0})$. Since

$$
V\left(H_{G_{\sigma}}\right) \cap\left(\mathbb{K}^{*}\right)^{n}=\pi^{-1}(V(I)) \cap U_{\sigma} \cap\left(\mathbb{K}^{*}\right)^{n},
$$

we have that

$$
\widetilde{V(I)}=V\left(H_{G_{\sigma}}\right)
$$

and the result is proved.

Theorem 13.2. Let $I \subset \mathbb{K}\left[x_{1}, \ldots, x_{n}\right]$ be an ideal, let $\Sigma$ be a regular refinement of the Gröbner fan $\Sigma(I)$, and let $\widehat{V(I)}$ be the strict transform of $V(I)$ under the modification given by $\Sigma$.

Given $\tau \in \Sigma$ we have

$$
\widetilde{V(I)} \cap \mathcal{O}(\tau) \neq \emptyset \Leftrightarrow \tau \subset \mathbf{T V}(I) .
$$

Proof. One implication is Proposition 12.1 .

Given $\tau \in \Sigma$, take $\sigma=\operatorname{Cone}(M) \in \Sigma$ of maximal dimension such that $\tau$ is generated by the first $s$ columns of $M$.

Let $G_{\sigma}$ be a system of generators as in Proposition 9.3 . Let $H_{G_{\sigma}}$ be as in 201. Take $y \in\left(\mathbb{K}^{*}\right)^{n}$ such that $I n_{\tau} g(y)=0$ for all $g \in G_{\sigma}$. 
For each $g \in G_{\sigma}$ write, the same way we did in 19 ,

$$
g \circ \phi_{M^{t}}=x^{\mathbf{P}_{g}} h_{g} \quad \text { with } \quad h_{g}(\underline{0}) \neq 0 .
$$

We have

$$
\widetilde{V(I)}=V\left(\left\{h_{g}\right\}_{g \in G_{\sigma}}\right)
$$

Set

$$
z=\left(z_{1}, \ldots, z_{n}\right)=\phi_{M^{t}}^{-1}(y),
$$

we have

$$
0=I n_{\tau} g(z)=z^{\mathbf{P}_{g}}\left(\left.h_{g}\right|_{\text {Cone }\left(e^{(s+1)}, \ldots, e^{(n)}\right)}\right)(z)
$$

then

$$
0=\left.h_{g}\right|_{\text {Cone }\left(e^{(s+1)}, \ldots, e^{(n)}\right)}(z)=h_{g}\left(0, \ldots, 0, z_{s+1}, \ldots, z_{n}\right) .
$$

Therefore, we conclude

$$
\left(0, \ldots, 0, z_{s+1}, \ldots, z_{n}\right) \in \mathcal{O}(\tau) \cap \widetilde{V(I)} .
$$

\section{References}

[AIL] Fuensanta Aroca, Giovanna Ilardi, and Lucía López de Medrano. Puiseux power series solutions for systems of equations. Internat. J. Math., 21(11):1439-1459, 2010.

[AHV] José M. Aroca, Heisuke Hironaka, and José L. Vicente. Desingularization theorems, volume 30 of Memorias de Matemática del Instituto "Jorge Juan" [Mathematical Memoirs of the Jorge Juan Institute]. Consejo Superior de Investigaciones Científicas, Madrid, 1977.

[BiGr] Robert Bieri and J. R. J. Groves. The geometry of the set of characters induced by valuations. J. Reine Angew. Math., 347:168-195, 1984.

[Biv] Carles Bivià-Ausina. Mixed Newton numbers and isolated complete intersection singularities. Proc. Lond. Math. Soc. (3), 94(3):749-771, 2007.

[BrNe] Gábor Braun and András Némethi. Invariants of Newton non-degenerate surface singularities. Compos. Math., 143(4):1003-1036, 2007.

[DGPS] Wolfram Decker, Gert-Martin Greuel, Gerhard Pfister, and Hans Schönemann. Singular 3.1.0 - A computer algebra system for polynomial computations., 2009. http://www.singular.uni-kl.de.

[Ehl] Fritz Ehlers. Eine Klasse komplexer Mannigfaltigkeiten und die Auflösung einiger isolierter Singularitäten. Math. Ann., 218(2):127-156, 1975.

[FJT] Komei Fukuda, Anders N. Jensen, and Rekha R. Thomas. Computing Gröbner fans. Math. Comp., 76(260):2189-2212 (electronic), 2007. 
[Ful] William Fulton. Introduction to toric varieties, volume 131 of Annals of Mathematics Studies. Princeton University Press, Princeton, NJ, 1993. The William H. Roever Lectures in Geometry.

[Gat] Andreas Gathmann. Tropical algebraic geometry. Jahresber. Deutsch. Math.-Verein., 108(1):3-32, 2006. arXiv:math.AG/0601322v1".

[Gom] Mirna Gómez-Morales. Resolución tórica de ideales Newton no degenerados. Master thesis, Universidad Nacional Autónoma de México, 2009.

[Hir] Heisuke Hironaka. Resolution of singularities of an algebraic variety over a field of characteristic zero. I, II. Ann. of Math. (2) 79 (1964), 109-203; ibid. (2), 79:205-326, 1964.

[IMS] Ilia Itenberg, Grigory Mikhalkin, and Eugenii Shustin. Tropical algebraic geometry, volume 35 of Oberwolfach Seminars. Birkhäuser Verlag, Basel, 2007.

[Jen] Anders Nedergaard Jensen. Gfan, a software system for Gröbner fans and tropical varieties., 2009. http://www.math.tu-berlin.de/ jensen/software/gfan/gfan.html.

[Kho1] Askold G. Khovanskii. Newton polyhedra and the genus of complete intersections. Funktsional. Anal. i Prilozhen., 12(1):51 - 61, 1977.

[Kho2] Askold G. Khovanskii. Newton polyhedra, and toroidal varieties. Funkcional. Anal. i Priložen., 11(4):56-64, 96, 1977.

[Kou] Anatoli G. Kouchnirenko. Polyèdres de Newton et nombres de Milnor. Invent. Math., 32(1):1-31, 1976.

[Mer] Michel Merle. Polyedre de Newton, eventail et desingularisation. (D'apres A. N. Varchenko). Semin. sur les singularites des surfaces, Cent. Math. Ec. Polytech., Palaiseau 1976-77, Lect. Notes Math. 777, 289-294 (1980)., 1980.

[MoRo] Teo Mora and Lorenzo Robbiano. The grbner fan of an ideal. J. Symbolic Comput., 6:183-208, 1988.

[Oka1] Mutsuo Oka. Principal zeta-function of nondegenerate complete intersection singularity. J. Fac. Sci. Univ. Tokyo Sect. IA Math., 37(1):11-32, 1990.

[Oka2] Mutsuo Oka. Non-degenerate complete intersection singularity. Actualités Mathématiques. [Current Mathematical Topics]. Hermann, Paris, 1997.

[RiSt] Jürgen Richter-Gebert, Bernd Sturmfels, and Thorsten Theobald. First steps in tropical geometry. In Idempotent mathematics and mathematical physics, volume 377 of Contemp. Math., pages 289-317. Amer. Math. Soc., Providence, RI, 2005. arXiv:math.AG/0306366. 
[SaZu] Marcelo J. Saia and Wilson A. Zuniga-Galindo. Local zeta function for curves, non-degeneracy conditions and Newton polygons. Trans. Amer. Math. Soc., 357(1):59-88 (electronic), 2005.

[Stu] Bernd Sturmfels. Gröbner bases and convex polytopes, volume 8 of University Lecture Series. American Mathematical Society, Providence, RI, 1996.

[Tei] Bernard Teissier. Monomial ideals, binomial ideals, polynomial ideals. In Trends in commutative algebra, volume 51 of Math. Sci. Res. Inst. Publ., pages 211-246. Cambridge Univ. Press, Cambridge, 2004.

[Tju] Galina Nikolaevna Tjurina. Absolute isolation of rational singularities, and triple rational points. Funkcional. Anal. i Priložen., 2(4):70-81, 1968.

[LeTo] Lê Dũng Tráng and Meral Tosun. Combinatorics of rational singularities. Comment. Math. Helv., 79(3):582-604, 2004. 